\title{
Influência do Método de Filetagem e Categorias de Peso sobre Rendimento de Carcaça, Filé e Pele da Tilápia do Nilo (Oreochromis niloticus)
}

\author{
Maria Luiza Rodrigues de Souza ${ }^{1}$, Elisabete M. Macedo-Viegas ${ }^{2}$, Sérgio do Nascimento Kronka ${ }^{3}$
}

\begin{abstract}
RESUMO - $O$ objetivo deste trabalho foi avaliar o efeito do método de filetagem $\left(\mathrm{F}_{1}=\right.$ retirada da pele com alicate e filetagem; $\mathrm{F}_{2}=$ filetagem e retirada da pele, com faca e auxílio de alicate) e a categoria de peso $\left(\mathrm{P}_{1}=250-300 \mathrm{~g} ; \mathrm{P}_{2}=301-350 \mathrm{~g} ; \mathrm{P}_{3}=351-400 \mathrm{~g}\right.$ e $\mathrm{P}_{4}=401-$ $450 \mathrm{~g}$ ) sobre o rendimento de carcaça (RC), filé (RF) e pele da tilápia do Nilo. Foram utilizadas 48 tilápias, em delineamento inteiramente casualizado. Houve efeito para método de filetagem, sendo a média de $\mathrm{F}_{1}\left(56,43\right.$ e 36,67\%) superior à de $\mathrm{F}_{2}$, 53,46 e 32,89\%, respectivamente, para RC e RF. Para categoria de peso, as médias $\mathrm{P}_{1}\left(56,49\right.$ e 37,34\%) e $\mathrm{P}_{2}(56,34$ e 36,40\%) foram superiores em relação a $\mathrm{P}_{3}(53,27$ e $31,98 \%)$ e $\mathrm{P}_{4}(53,71$ e $33,42 \%)$, respectivamente, para RC e RF. Porcentagens de pele bruta, limpa e de descarne foram superiores para $\mathrm{F}_{2}$, mas não houve efeito para as categorias de peso. A filetagem $\mathrm{F}_{1}$ proporcionou melhores rendimentos e resultados de pele e, para categoria de peso, $\mathrm{P}_{1}$ e $\mathrm{P}_{2}$, maiores rendimentos.
\end{abstract}

Palavras-chave: Oreochromis niloticus, produção de pescado, processamento de pescado, processamento de filetagem, Tilápia do Nilo

\section{Influence of Processing Method and Weight Categories on Carcass, Fillet and Skin Yield of Nile Tilapia (Oreochromis niloticus)}

\begin{abstract}
The objective of this study was to evaluate the processing methods $\left(\mathrm{F}_{1}=\right.$ to remove skin with pliers and then to cut in fillets; $F_{2}=$ to cut in fillet and then to remove skin with knife and pliers help) and weight categories $\left(\mathrm{W}_{1}=250-300 \mathrm{~g} ; \mathrm{W}_{2}=301-350 \mathrm{~g}\right.$; $\mathrm{W}_{3}=351-400 \mathrm{~g}$ and $\mathrm{W}_{4}=401-450 \mathrm{~g}$ ), on the carcass (CY), fillet (FY) and skin yield of Nile tilapia. Forty-eight fishes were used in a completely randomized design. There was effect for the processing method, being the $\mathrm{F}_{1}$ mean $(56.43$ and $36.67 \%)$ higher to the $\mathrm{F}_{2}(53.46$ and $32.89 \%)$ for CY and FY respectively. For the weight categories, $\mathrm{W}_{1}(56.49$ and $37.34 \%)$ and $\mathrm{W}_{2}(56.34$ and $36.40 \%)$ were superior as compared to $\mathrm{W}_{3}(53.27$ and $31.98 \%)$ and $\mathrm{W}_{4}(53.71$ and $33.42 \%)$, respectively for CY and FY. Crude skin percentage, clean and of fleshed were higher for $\mathrm{F}_{2}$, but there was no effect for weight categories. The $\mathrm{F}_{1}$ processing method promoted the best yield and skin results, and for the weight categories $\mathrm{W}_{1}$ and $\mathrm{W}_{2}$ higher yields.
\end{abstract}

Key Words: Oreochromis niloticus, fish production, fish processing, fillet processing, Nile Tilapia

\section{Introdução}

A produção de alimento de qualidade para o consumo humano, uma das prioridades dos pesquisadores do setor agropecuário, destaca a participação da aqüicultura, bem como o seu potencial na produção mundial de alimentos protéicos de origem animal. Segundo TACON (1996), esta é a atividade zootécnica com maior índice de crescimento nesta década, cerca de $10,4 \%$ ao ano.

Dentro da aqüicultura, a piscicultura de água doce é a atividade que vem se mostrando mais promissora, sendo a tilápia do Nilo uma das espécies mais utilizadas mundialmente (CLEMENT e LOVELL, 1994), com maior potencial de cultivo. A demanda de consumo de seu filé tem crescido subs- tancialmente nos últimos anos, sendo uma das espécies mais indicadas para o cultivo intensivo, devido a suas qualidades para a produção, excelente textura e paladar da sua carne, por não apresentar microespinhas e possibilitar a filetagem e a industrialização da carcaça (SCHMIDT, 1988; BOLL et al., 1995). Além destas qualidades, pode-se aliar a possibilidade do uso de sua pele para o curtimento, que pode representar outra fonte de renda para o piscicultor ou para a indústria de filetagem.

A definição do peso de abate, os diferentes métodos de processamento, o conhecimento sobre o rendimento do peixe, bem como os seus subprodutos, são de fundamental importância tanto para a indústria de processamento como para o produtor.

Para a produção da tilápia, devem ser considera-

\footnotetext{
1 Prof. do Departamento de Zootecnia da Universidade Estadual de Maringá/UEM. Av. Colombo 5790 - 87020-900, Maringá - PR, Brasil.

2 Prof. do Centro de Aqüicultura da Universidade Estadual Paulista/UNESP. Rodovia Carlos Tonanni, km 5 - 14870 -000 - Jaboticabal - SP, Brasil.

3 Pesquisador do CNPq. Prof. do Centro de Aqüicultura da Universidade Estadual Paulista/UNESP. Rodovia Carlos Tonanni, km 5 -14870-000 Jaboticabal - SP, Brasil.
} 
das algumas características mercadológicas, como o tamanho ou peso do peixe para o abate e peso do filé, por proporcionar maior rendimento de carcaça e de filé. Apesar de o rendimento de filé depender da eficiência das máquinas filetadoras, destreza manual do operário e da forma anatômica do corpo do peixe (CONTRERAS-GUZMÁN, 1994), deve-se considerar também a forma pela qual é realizada a esfola, ou seja, a retirada da pele da carcaça ou do filé.

Tendo em vista o crescente interesse pelo cultivo, pela industrialização e pela boa aceitação do filé de tilápia, aliado à carência de dados sobre seu processamento, realizou-se este trabalho com o objetivo de avaliar os rendimentos de carcaça e filé e as porcentagens da pele bruta, limpa e de descarne, da tilápia do Nilo, submetida a dois métodos de filetagem manual, para quatro categorias de peso.

\section{Material e Métodos}

\section{Animais experimentais e procedimentos de filetagem}

Oexperimento foi realizado no Centro de Aqüicultura da UNESP (CAUNESP-Jaboticabal), no mês de fevereiro de 1997. Foram utilizados 48 exemplares de tilápia do Nilo (Oreochromis niloticus), capturados de tanques de $38 \mathrm{~m}^{2}$, por meio de tarrafas e sacrificados por destruição da medula espinhal.

Após o sacrifício, cada exemplar foi eviscerado e filetado, sendo submetido às pesagens em balança de precisão de $0,01 \mathrm{~g}$ e às medições de comprimento padrão (ponta do focinho até o pendúnculo caudal) e altura, com auxílio de um ictiômetro (graduado em $\mathrm{cm})$. Consideraram-se o peso total, a carcaça (eviscerada, sem cabeça e pele), o filé sem pele e o peso da pele bruta (após a esfola, com escamas e restos de músculos) e limpa (após escamar e descarnar), para se determinarem o rendimento de carcaça (RC), o rendimento de filé (RF) e a porcentagem da pele bruta (PPB), limpa (PPL) e de descarne (PD).

O processo de filetagem foi realizado por uma única pessoa, aplicando-se os métodos de filetagem, ou seja, o método $\mathrm{F}_{1}$, retirando-se a pele com alicate de ponta fina e depois o filé (Figura 1) e o outro método, o $\mathrm{F}_{2}$, obtendo-se primeiramente o filé com pele e, em seguida, separando-se esta do filé com a faca e o auxílio do alicate de ponta fina (Figura 2), relatado por FREITAS e GURGEL (1984). O filé foi obtido a partir da musculatura dorsal nas duas laterais do peixe no sentido longitudinal, ao longo de toda a extensão da coluna vertebral e costelas. Todos os dados de rendimento foram calculados em função do peso total do exemplar.

\section{Delineamento experimental}

$\mathrm{O}$ delineamento experimental foi inteiramente casualizado, com oito tratamentos, em fatorial $2 \times 4$, constituído de dois métodos de filetagem $\left(\mathrm{F}_{1}=\right.$ retirada da pele e filetagem; $F_{2}=$ filetagem com a pele e retirada da pele $)$ e quatro categorias de peso $\left(\mathrm{P}_{1}=250\right.$ $300 \mathrm{~g} ; \mathrm{P}_{2}=301-350 \mathrm{~g} ; \mathrm{P}_{3}=351-400 \mathrm{~g} \mathrm{e}_{4}=401-450$ g), com seis repetições por tratamento, em que cada peixe foi considerado uma parcela experimental. Os dados de rendimento de carcaça, filé, porcentagem da pele bruta, limpa e descarne foram submetidos à análise de variância e as médias foram comparadas pelo teste Tukey, a 5\% de probabilidade (BANZATTO e KRONKA, 1995). O modelo matemático utilizado foi:

$$
\mathrm{Y}_{\mathrm{ijk}}=\mu+\mathrm{F}_{\mathrm{i}}+\mathrm{P}_{\mathrm{j}}+(\mathrm{FP})_{\mathrm{ij}}+\mathrm{e}_{\mathrm{ijk}}
$$

em que

$\mathrm{Y}_{\mathrm{ijk}}=$ valor observado para o peixe $\mathrm{k}$, com método de filetagem i e categoria de peso $\mathrm{j}$;

$\mu=$ média;

$\mathrm{F}_{\mathrm{i}}=$ efeito do método de filetagem $\mathrm{i}(\mathrm{i}=1,2)$;

$\mathrm{P}_{\mathrm{j}}=$ efeito da categoria de peso $\mathrm{j}(\mathrm{j}=1,2,3,4)$;

$(\mathrm{FP})_{\mathrm{ij}}=$ efeito da interação do método de filetagem i e a categoria de peso $\mathrm{j}$; e

$\mathrm{e}_{\mathrm{ijk}}=$ erro.

\section{Resultados e Discussão}

No Brasil são poucos os estudos relativos ao rendimento de carcaça ou filé de peixes. Em geral, não existe padronização no método de filetagem e na categoria de peso, em função das espécies de peixes sobre os seus rendimentos. Assim, os peixes foram capturados e classificados nas quatro categorias de peso, em função do que mais se utiliza em indústrias filetadoras e comercialização pelo piscicultor. Na Tabela 1, constam os valores médios do comprimento padrão e da altura (cm) dos peixes utilizados para análise do método de filetagem dentro de cada categoria de peso.

Comparando-se o método de filetagem para o rendimento de carcaça e filé (Tabela 2), houve efeito significativo $(\mathrm{P}<0,01)$, sendo a média da filetagem $\mathrm{F}_{1}$ $(56,43$ e $36,67 \%)$ superior à da filetagem $\mathrm{F}_{2}(53,46 \mathrm{e}$ $32,89 \%$ ), respectivamente, para as características analisadas. Pode-se observar que retirando o filé e, em seguida, a pele $\left(\mathrm{F}_{2}\right)$, permanece nesta quantidade razoável de músculos, proporcionando menor rendimento de filé, enquanto, retirando a pele e depois a filetagem $\left(\mathrm{F}_{1}\right)$, evidencia-se menor perda de carcaça e filé. 


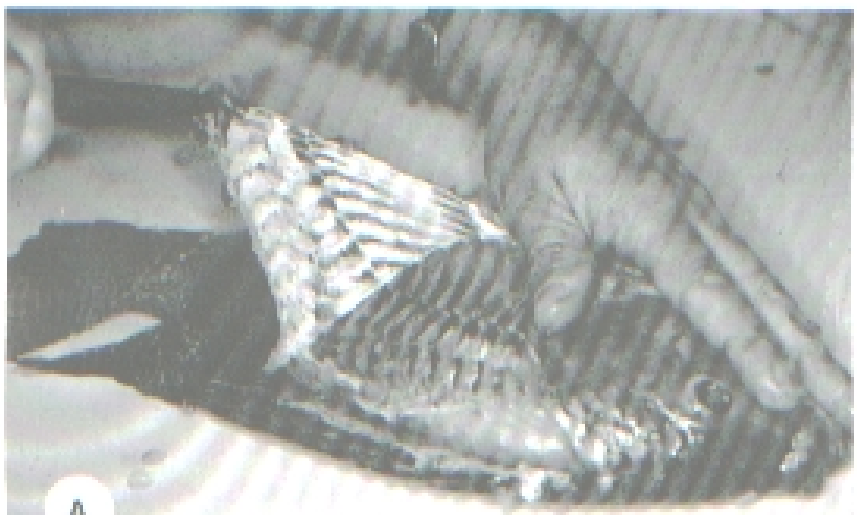

A

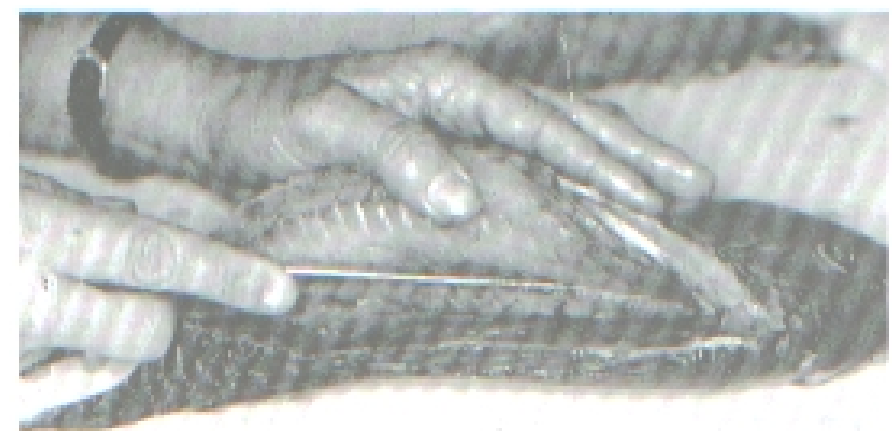

B

Figura 1 - Método de filetagem $F_{1}$ : retirada da pele com auxílio de alicate $(A)$ e depois o filé $(B)$.

Figure 1 - Processing method $F_{1}$ : to remove skin with pliers $(A)$ and then to cut in fillets $(B)$
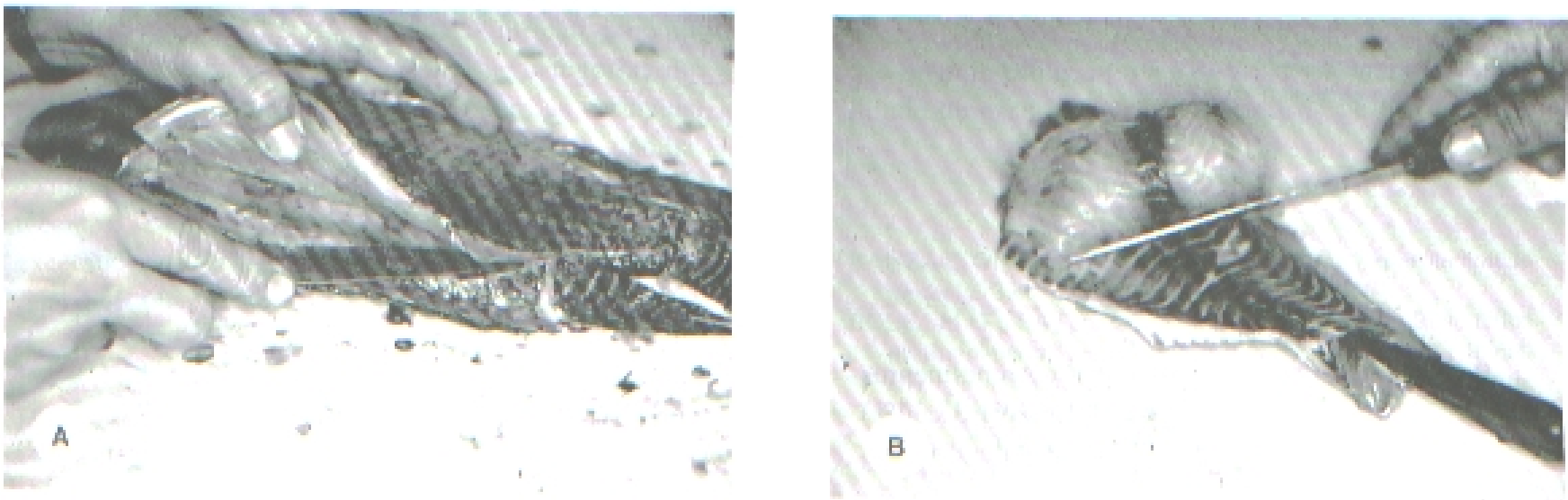

Figura 2 - Método de filetagem $\mathrm{F}_{2}$ : retirada do filé com pele $(A)$ e depois a retirada da pele, com faca e auxílio do alicate Figure 2 - Processing method $F_{2}$ : to cut in skin with fillet $(A)$ and then to remove skin with knife and pliers. 
SOUZA et al.

Tabela 1 - Dados biométricos médios de exemplares de Tilápia do Nilo

Table 1 - Average biometric data of specimens of Nile Tilapia

\begin{tabular}{lcc}
\hline Categoria de peso $(\mathrm{P})$ & $\begin{array}{c}\text { Comprimento padrão }(\mathrm{cm}) \\
\text { Weight categorie }(W)\end{array}$ & $\begin{array}{c}\text { Altura }(\mathrm{cm}) \\
\text { Standard length }\end{array}$ \\
\hline $\mathrm{P}_{1}=250-300 \mathrm{~g}$ & 19,62 & 7,48 \\
$\mathrm{P}_{2}=301-350 \mathrm{~g}$ & 20,56 & 8,06 \\
$\mathrm{P}_{3}=351-400 \mathrm{~g}$ & 21,10 & 8,60 \\
$\mathrm{P}_{4}=401-450 \mathrm{~g}$ & 21,65 & 9,13 \\
\hline
\end{tabular}

Tabela 2 - Valores médios de rendimento de carcaça, filé e porcentagens de pele bruta, limpa e de descarne da Tilápia do Nilo Table 2 - Mean values of the carcass, fillet yield and crude skin percentage, clean and of fleshed of Nile Tilapia

Fonte de variação

Source of variation

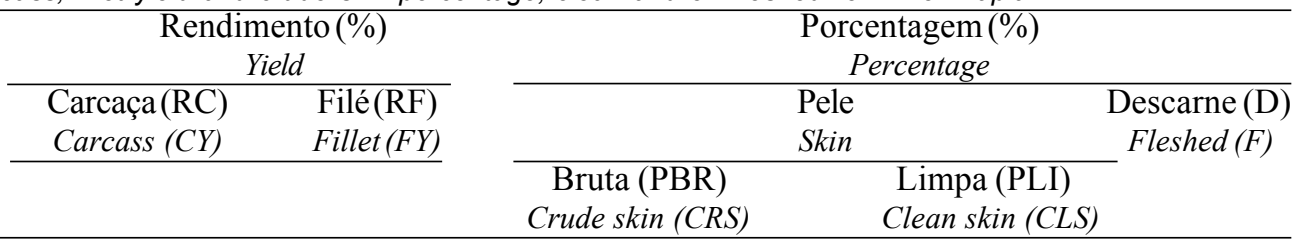

Filetagem(F)

Crude skin (CRS)

$\begin{array}{lllll}56,43 \mathrm{a} & 36,67 \mathrm{a} & 5,32 \mathrm{~b} & 3,14 \mathrm{~b} & 40,63 \mathrm{~b}\end{array}$

$\mathrm{F}_{1}=$ retirar pele e filetar

$3,45 \mathrm{a}$

$58,97 \mathrm{a}$

$\mathrm{F}_{2}=$ filetar e retirar pele

$\mathrm{F}_{2}=$ to cut in fillet and to remove skin

$53,46 \mathrm{~b}$

$32,89 b$

$8,51 \mathrm{a}$

$3,45 \mathrm{a}$

Weight categories $(W)$

$\mathrm{P}_{1}=250-300 \mathrm{~g}$

$\mathrm{P}_{2}=301-350 \mathrm{~g}$

$\mathrm{P}_{3}=351-400 \mathrm{~g}$

$\mathrm{P}_{4}=401-450 \mathrm{~g}$

Teste $\mathrm{F}$

Ftest

Filetagem(F)

Fillet

Categorias de peso (P)

Weight categories (W)

Interação (F x P)

Interaction $(F \times W)$

\begin{tabular}{lll}
$56,49 \mathrm{a}^{(1)}$ & $37,34 \mathrm{a}$ & $6,83 \mathrm{a}$ \\
$56,34 \mathrm{ab}$ & $36,40 \mathrm{ab}$ & $7,09 \mathrm{a}$ \\
$53,27 \mathrm{c}$ & $31,98 \mathrm{c}$ & $6,81 \mathrm{a}$ \\
$53,71 \mathrm{bc}$ & $33,42 \mathrm{bc}$ & $6,92 \mathrm{a}$ \\
\hline
\end{tabular}

\begin{tabular}{ll}
$3,32 \mathrm{a}$ & $50,01 \mathrm{a}$ \\
$3,37 \mathrm{a}$ & $50,16 \mathrm{a}$ \\
$3,33 \mathrm{a}$ & $47,08 \mathrm{a}$ \\
$3,15 \mathrm{a}$ & $51,95 \mathrm{a}$ \\
\hline
\end{tabular}

$\mathrm{CV}(\%)$

$17,04 * *$

$5,58 *$

1,03

4,54

1 Médias na coluna, para cada fator, seguidas de letras diferentes são diferentes $(P<0,05)$ teste Tukey.

Means, within a column, by each factor, followed different letters are diferent $(P<.05)$ by Tukey test.

* Significativo [significant] $(\mathrm{P}<0,05)$.

** Significativo [significant] $(\mathrm{P}<0,01)$.

Para as categorias de peso, os maiores rendimentos de carcaça e filé foram obtidos em $\mathrm{P}_{1}(56,49$ e $37,34 \%)$ e $\mathrm{P}_{2}(56,34$ e $36,40 \%)$, em relação às categorias $\mathrm{P}_{3}$ $(53,27$ e $31,98 \%)$ e $\mathrm{P}_{4}(53,71$ e $33,42 \%)$, respectivamente, para carcaça e filé. Houve diferença significativa entre a média de $\mathrm{P}_{1}(250$ - $300 \mathrm{~g})$ em relação à de $\mathrm{P}_{3}(351-400 \mathrm{~g})$ e $\mathrm{P}_{4}(401-450 \mathrm{~g})$ e entre a média de $\mathrm{P}_{2}$ (301 - $\left.350 \mathrm{~g}\right)$ e $\mathrm{P}_{3}(351$ - $400 \mathrm{~g})$ (Tabela 2).

Segundo CONTRERAS-GUZMÁN (1994), a carcaça, em média, é $62,6 \%$ do peso dos peixes. Para a tilápia do Nilo pesando $585 \mathrm{~g}$, considerando a carcaça sem cabeça, pele e vísceras. CLEMENT e LOVELL (1994) obtiveram valor de rendimento de carcaça de $51 \%$, o qual é inferior aos obtidos neste experimento com peixes de 250 a 450 g $(53,27$ a $56,49 \%$ ), conforme Tabela 2. NOVATO e VIEGAS (1997), trabalhando com a tilápia vermelha da Flórida, com peso de 451 a $550 \mathrm{~g}$, verificaram rendimento de 
carcaça sem cabeça de 75,5\%.

Stansby e Olcott (1963), citados por FREITAS et al. (1979), afirmaram que o rendimento de filé varia de acordo com a espécie, entre 20 a $40 \%$, enquanto SANTOS et al. (1995) mencionaram rendimento de filé de $21 \%$ para o cascudo; para o bagre africano, HOFFMAN et al. (1993) obtiveram 38,9 a 46,7\%; POUEY e STINGELIN (1996), 44\% para o peixe-rei.

CONTRERAS-GUZMÁN (1994) cita que a tilápia está entre as espécies que apresenta o menor rendimento de filé (inferiores a $40 \%$ ); segundo o mesmo autor, rendimentos obtidos por Gurgel e Freitas (1972) e Freitas et al. (1972), para tilápias pesando de 400 a 600 $\mathrm{g}$ de peso vivo, foram acima de $32 \%$. SOUZA et al. (1997) também citam rendimentos de filé de 35,8 a $37,2 \%$, para tilápia do Nilo. Todavia, MACEDO-VIEGAS etal.(1997) obtiveram porcentagens de 32,15 a 40,39\%, para a mesma espécie, pesando de 250 a 450 g. Entretanto, CLEMENT eLOVELL (1994) relataram rendimento de filé de 25,4\% para a tilápia do Nilo, com peso médio de $585 \mathrm{~g}$.

Portanto, os resultados obtidos neste experimento estão de acordo com os citados por CONTRERASGUZMÁN (1994), inferiores aos citados por MACEDO-VIEGAS et al. (1997) e superiores ao relatado por CLEMENT e LOVELL (1994). Esta variação pode estar relacionada à forma de filetagem, ou seja, o método utilizado para a filetagem, que nem sempre é bem descrito, no trabalho; à experiência do filetador (mão-de-obra); ao estádio de desenvolvimento gonadal (reprodutivo); e à faixa de peso em que os peixes se encontravam na fase de abate.

As porcentagens de pele bruta, limpa e de descarne sofreram o efeito do procedimento de filetagem, em que as médias da filetagem $F_{2}$ foram significativamente superiores às da filetagem $\mathrm{F}_{1}$. Para as categorias de peso, não houve efeito significativo para as variáveis estudadas (Tabela 2). Na literatura consultada, não há dados relativos a estas variáveis, pelo fato de ser recente o interesse pelo curtimento de peles de peixes, exceto alguns dados referentes a peso ou à porcentagem de pele de determinadas espécies de peixes.

Segundo CONTRERAS-GUZMÁN (1994), a pele perfaz, em média, 7,5\% do peso dos peixes teleósteos. FREITAS et al. (1979) e FREITAS e GURGEL (1984) citaram que a pele da tilápia do Nilo, sem escama, corresponde a 5,0\% do peso do peixe inteiro. De acordo com MACEDO-VIEGAS et al. (1997), a pele bruta da tilápia do Nilo, com pesos entre 250 a 450 $\mathrm{g}$, utilizando o método de filetagem descrito neste trabalho como $\mathrm{F}_{1}$, ou seja, retirou-se primeiramente a pele do peixe com auxílio de um alicate com ponta fina e, em seguida, a obtenção dos filés, foi de 4,77 a 5,71\%. Entretanto, os valores obtidos por MACEDO-VIEGAS et al. (1997) foram inferiores aos obtidos neste experimento, para as mesmas categorias de peso.

Para a indústria coureira que paga por peso, ou para o produtor que processa a pele, é interessante que as mesmas apresentem menor quantidade de escamas e musculatura, pois facilita o curtimento, devido às menores quantidades de produtos químicos necessários e mão-de-obra (tempo para descarnar a pele), o que é proporcionado pelo método de filetagem $F_{1}$. No método de filetagem $F_{2}$, observa-se que a porcentagem de pele limpa é superior à filetagem $\mathrm{F}_{1}$, em virtude das dificuldades de remoção de toda a musculatura e gordura da pele no método $\mathrm{F}_{2}$, permanecendo ainda alguns desses resíduos na própria pele.

Quanto ao descarne, o método de filetagem $\mathrm{F}_{2}$ (Figura 2) foi significativamente superior $(58,97 \%)$ ao método $\mathrm{F}_{1}(40,63 \%)$. Portanto, a filetagem $\mathrm{F}_{2}$ proporcionou maior quantidade de resíduos (músculos e gordura) para ser retirada das peles em relação ao método $F_{1}$. Estes resíduos são prejudiciais ao processamento das peles, ou seja, para o curtimento. Para execução do curtimento das peles, estes resíduos devem ser totalmente retirados, caso contrário, poderá proporcionar peles mal curtidas e endurecidas.

\section{Conclusões}

O método de se retirar a pele com alicate e depois o filé proporcionou maior rendimento de carcaça e filé. Os peixes menores (250-300 g e 301-350 g) apresentaram os melhores rendimentos de carcaça e filé. A filetagem $\mathrm{F}_{1}$ proporcionou melhores resultados de pele bruta, limpa e de descarne (menor quantidade de escamas e musculatura na pele).

\section{Referências Bibliográficas}

BANZATTO, D.A., KRONKA, S.N. 1995. Experimentação agrícola. 3.ed. Jaboticabal: FUNEP. 247p.

BOLL, M.G., SATO, G., AMARAL JR., H.A. Resultados preliminares de método alternativo de manejo da T ilápia, Oreochromis niloticus, no período de inverno em regiões de clima subtropical. In: ENCONTRO RIOGRANDENSE DE TÉCNICOS EM AQÜICULTURA, 6, ENCONTRO BRASILEIRO DE AQÜICULTURA, 3, 1995, Ibirubá. Anais... Porto Alegre: Universidade Federal do Rio Grande do Sul, Departamento de Zootecnia, Setor de Aqüicultura. 1995. p.88-93.

CLEMENT, S., LOVELL, R.T. 1994. Comparison of processing yield and nutrient composition of culture Nile tilapia (Oreochromis niloticus) and channel catfish (Ictalurus punctatus). Aquaculture, 119:299-310.

CONTRERAS-GUZMÁN, E.S. Bioquímica de pescados e deri- 
vados. Jaboticabal: FUNEP, 1994, 409p.

FREITAS, J.V.F., GURGEL, J.J.S. 1984. Estudos experimentais sobre a conservação da tilápia do Nilo, Oreochromis niloticus (L. 1766) Trewavas, armazenada no gelo. Bol. Téc. DNOCS, 42(2):153-178.

FREITAS, J.V.F., GURGEL, J.J.S., MACHADO, Z.L. 1979. Estudos de alguns parâmetros biométricos e da composição química, inclusive sua variação sazonal, da tilápia do Nilo, Sarotherodon niloticus (L.) do açude público "Paulo Sarasate" (Reriutaba, Ceará, Brasil), durante os anos de 1978 e 1979. Bol. Téc. DNOCS, 37(2):135-151.

HOFFMAN, L.C., CASEY, N.H., PRINSLOO, J.F. Carcass yield and fillet chemical composition of wild and farmed African sharptooth catfish, Clarias gariepinus. Int. Conf. Bordeaux Aquaculture, Bordeaux (France), 25-27, mar, 1992. Bordeaux, France: European Aquaculture Society, 1993. p.421-432. (Special Publications of European Aquaculture, n.18).

MACEDO-VIEGAS, E.M., SOUZA, M.L.R., KRONKA, S.N. 1997. Estudo da carcaça de tilápia do Nilo (Oreochromis niloticus), em quatro categorias de peso. Rev. UNIMAR, 19(3):863-870.

NOVATO, P.F.E., VIEGAS, E.M.M. Carcass yield analyses of Florida Red Tilapia in three weight classes. In: INTERNATIONAL SYMPOSIUM BIOLOGY OF TROPICAL FISHES, 1997. Manaus. Abstracts... Manaus: INPA. 1997. p. 150.

POUEY, J.F., STINGELIN, L.A. Rendimento da carcaça e da carne do peixe-rei (Odontesthes humensis), na faixa de 200 a 300 g. In: SIMPÓSIO BRASILEIRO DE AQÜICULTURA, 9, 1996, Sete Lagoas. Resumos... Sete Lagoas: ABRAq, 1996. p.141.
SANTOS, A.B., MELO, J.F.B., LOPES, P.R.S. Estudo da carcaça do cascudo Hypostomus commersonii na região de Uruguaiana-RS/ Brasil. In: ENCONTRO SUL BRASILEIRO DE AQÜICULTURA, 3. ENCONTRO RIOGRANDENSE DE TÉCNICOS EM AQÜICULTURA, 6, 1995, Ibirubá. Anais... Porto Alegre: Universidade Federal do Rio Grande do Sul, Departamento de Zootecnia, Setor de Aqüicultura. 1995. p.70-76.

SCHMIDT, A. A. P. 1988. Piscicultura:a fonte divertida de proteínas. São Paulo:Icone. 88p.

SOUZA, M.L.R., CASTAGNOLLI, N., KRONKA, S.N. Nile tilapia's carcass characteristics dependence on stocking density and aeration system. In: THE ANNUAL INTERNATIONAL CONFERENCE. EXPOSITION OF THE WORLD AQUACULTURE SOCIETY, Seattle. Abstracts... Seattle: World Aquaculture Society. 1997. p.398.

TACON, A.G.J. 1996. Trends in aquaculture production with particular reference to low income food deficit Countries 1984-1993. Food Aquaculture Newsletter, 12:6-9.

Recebido em: 12/03/98

Aceito em: 09/09/98 